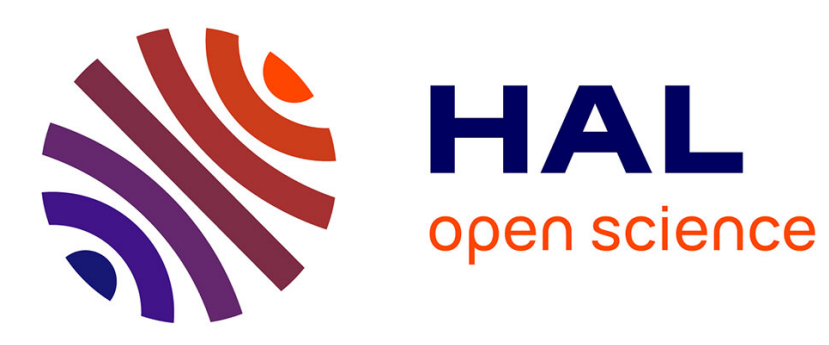

\title{
Dynamics of plasma gratings in atomic and molecular gases
}

\author{
Magali Durand, Amélie Jarnac, Yi Liu, Bernard Prade, Aurélien Houard, \\ Vladimir Tikhonchuk, André Mysyrowicz
}

\section{- To cite this version:}

Magali Durand, Amélie Jarnac, Yi Liu, Bernard Prade, Aurélien Houard, et al.. Dynamics of plasma gratings in atomic and molecular gases. Physical Review E , 2012, 86 (3), pp.036405. 10.1103/PhysRevE.86.036405 . hal-00852038

\section{HAL Id: hal-00852038 \\ https://hal-polytechnique.archives-ouvertes.fr/hal-00852038}

Submitted on 19 Aug 2013

HAL is a multi-disciplinary open access archive for the deposit and dissemination of scientific research documents, whether they are published or not. The documents may come from teaching and research institutions in France or abroad, or from public or private research centers.
L'archive ouverte pluridisciplinaire HAL, est destinée au dépôt et à la diffusion de documents scientifiques de niveau recherche, publiés ou non, émanant des établissements d'enseignement et de recherche français ou étrangers, des laboratoires publics ou privés. 


\title{
Dynamics of plasma gratings in atomic and molecular gases
}

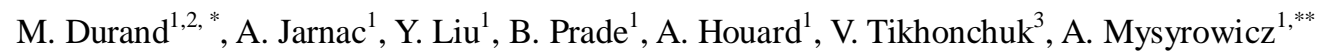 \\ ${ }^{1}$ Laboratoire d'Optique Appliquée, ENSTA ParisTech, Ecole Polytechnique, CNRS, 91761, Palaiseau, France \\ ${ }^{2}$ Departement d'Optique Théorique et Appliquée, ONERA, 91123, Palaiseau, France \\ ${ }^{3}$ Univ. Bordeaux, CNRS, CEA, CELIA (Centre Lasers Intenses et Applications) UMR 5107, 33405, Talence, France
}

\begin{abstract}
The decay of the plasma grating formed at the intersection of two femtosecond filaments is measured in several molecular and atomic gases. The grating evolution is ruled by ambipolar diffusion in atomic gases and by a combination of ambipolar diffusion and collision-assisted free electron recombination in molecular gases. Electron diffusion and recombination coefficients are extracted for $\mathrm{Ne}, \mathrm{Ar}, \mathrm{Kr}, \mathrm{Xe}, \mathrm{N}_{2}, \mathrm{O}_{2}, \mathrm{CO}_{2}$ and air at 1 bar.
\end{abstract}

\footnotetext{
* Now at Townes Laser Institute, CREOL - Orlando, FL 32816, USA

**andre.mysyrowicz@ensta.fr
} 
Intense femtosecond laser pulses propagating in a neutral gas undergo a beam collapse if the incident pulse peak power exceeds a critical value, $P_{c r} \approx 0.15 \lambda^{2} / n_{0} n_{2}$, where $n_{0}$ is the linear refractive index, $n_{2}$ the nonlinear index coefficient and $\lambda$ the wavelength of the laser pulse in vacuum $[1,2]$. The collapse is arrested by the defocusing effect due to multiphoton ionization of the gas. The ensuing dynamic competition between beam self focusing and plasma defocusing gives rise to filamentation, a propagation regime characterized by a contracted pulse keeping a high intensity over long distances, leaving a thin weakly ionized plasma column in its wake [3]. Recently, much attention has been given to the interaction between two crossing filaments [4-8]. Because of field interference, a two-dimensional plasma grating is formed in the intersection region when two non collinear filamentary pulses overlap in time. This grating plays an important role in the interaction between laser beams. For instance, the moving plasma grating formed by two intersecting filaments of slightly different central frequency is responsible for an important exchange of energy between the filaments [5]. A stationary grating formed with pulses of same frequency can interrupt the progression of a filament [9] or it can redirect a third beam of different frequency [7, 10]. Since the plasma grating persists well after the passage of the pulses, it provides a way to manipulate additional probe beams or filaments in a radiation free environment. The grating is periodic but anharmonic as the electron density created by the ionization process is a nonlinear function of the laser intensity. It is therefore important to measure its lifetime and understand the origin of its decay.

Recently, Shi et al. [11] have reported an exponential decay of the plasma grating formed in air by two UV filaments, with a lifetime on the order of 100 ps in air. However, as shown in this letter, the decay process cannot be expressed in most cases with a simple exponential law. In particular, the grating decay time may vary considerably in a given gas, depending on the grating fringe separation. Two distinct processes contribute to the grating decay: ambipolar diffusion and electron recombination. They have quite different characteristic times and the laser beam crossing experiment allows assessing these processes in a parameter domain that has never been explored so far. 
We have measured the plasma grating decay in several atomic and molecular gases at normal pressure. In atomic gases, we observe a decay that is dominated by diffusion. In molecular gases, both plasma recombination and diffusion contribute to the grating decay on a comparable time scale. From the measurements, we extract the coefficients of ambipolar diffusion and free electron recombination in $\mathrm{Ne}, \mathrm{Kr}$, $\mathrm{Ar}, \mathrm{Xe}$ and $\mathrm{N}_{2}, \mathrm{O}_{2}, \mathrm{CO}_{2}$, and air at atmospheric pressure.

The principle of the experiments is as follows (Fig. 1): In a first configuration A, two laser pulses at same frequency $\omega$ enter a cell containing the gas at atmospheric pressure where they form two filaments that propagate in the $y-z$ plane and intersect in the middle of the cell. A plasma grating is formed in the intersection region, as evidenced by the image of the plasma luminescence shown for air in Fig. 1(a). The main period of the observed fringe separation obeys well the relation $\Lambda=\lambda /(2 \sin (\Phi / 2))$, where $\Phi$ is the crossing angle. A weaker third probe beam at frequency $2 \omega$, collinear with one of the filament forming pulse, is diffracted by the grating in the direction of the other filament. In order to satisfy the Bragg condition, the probe beam is diffracted by the second order anharmonic term of the grating profile $\Lambda_{2}=\Lambda / 2$. The diffracted signal is measured as a function of the delay between the grating forming pulses and the probe for three different crossing angles, $\Phi=7^{\circ}, 14^{\circ}$ and $90^{\circ}$. Both filamentary pulses $(\lambda=800 \mathrm{~nm}$, duration 35 fs, pulse energy $1 \mathrm{~mJ}$ ) are derived from the same CPA Ti:Sa laser and are linearly polarized along $x$. The probe pulse (wavelength $400 \mathrm{~nm}$, duration $35 \mathrm{fs}$, pulse energy $30 \mu \mathrm{J}$ ), of same polarization, is obtained by frequency doubling in a BBO crystal of a fraction of the CPA laser output.

In a complementary experiment (configuration B), a collimated probe pulse at frequency $\omega$ propagates perpendicularly to the bi-dimensional fringe pattern formed by the two filaments crossing under angle $\Phi=7^{\circ}$ (see Fig. 1(b)). In this in-line holographic imaging technique [12-14], the diffraction pattern due to the plasma bubble is recorded at a distance of $38 \mathrm{~cm}$ from the grating by a CD camera as a function of the probe pulse delay. Here, the probe beam records the average free electron density through the concomitant 
variation of the refractive index. As the direction of the probe beam is parallel to the grating wavevector, there is no probe diffraction from the grating but only from the overall plasma filament.

Figure 2 shows the amplitude of the diffracted probe signal seen in configuration A as a function of time in the case of argon and $\mathrm{O}_{2}$ for three angles $\Phi[15,16]$. The same experiment has also been performed in $\mathrm{Ne}, \mathrm{Kr}, \mathrm{Xe}, \mathrm{N}_{2}, \mathrm{CO}_{2}$ and air. In all atomic gases, there is a strong dependence of the signal diffracted by the grating with angle $\Phi$, but no significant decay of the plasma during the same time interval. By contrast, in molecular gases, a rapid decay of the plasma is measured, as shown for $\mathrm{O}_{2}$ in Fig. 3 (b). The strong dependence of the signal with $\Phi$ in Fig. 2 (a) can be qualitatively explained by the fact that the grating fringe spacing decreases with increasing angle $\Phi$. Closer fringe spacing leads to a reduction of the time necessary for a diffusive wash out of the fringe pattern.

The electron diffusion in a weakly ionized plasma is strongly depending on the relation between the electron Debye length, $\lambda_{\mathrm{D}}$, electron mean free path, $\lambda_{\mathrm{en}}$, and the grating period, $\Lambda$. For the expected conditions in the plasma filament (the electron temperature is of the order or less than $1 \mathrm{eV}$ and the electron density $\sim 10^{18} \mathrm{~cm}^{-3}$ ) the electron Debye length, $\lambda_{D} \approx 0.07 \mu \mathrm{m}$, and the electron mean free path, $\lambda_{\text {en }} \approx$ $0.15 \mu \mathrm{m}$, are much smaller than the grating period $\Lambda$. Then a quasineutrality is maintained across the grating and the electron's diffusion is controlled by the ion mobility. As the ion temperature $T_{i}$ is close to room temperature and is much smaller than the electron temperature, Te, the ambipolar diffusion coefficient reads as $D_{a m} \approx D_{i}\left(1+T_{e} / T_{i}\right)$, where $D_{i}=k T_{i} \mu_{i} / e$ is the ion diffusion coefficient, $k$ is the Boltzmann constant, $\mu_{i}$ is the ion mobility and $e$ is the elementary charge.

Plasma recombination can occur through collision-assisted electron-ion recombination and additionally through dissociative recombination in molecules. The dissociative recombination process corresponds to the collision of an electron with a molecular ion that results in two neutral atoms at the output, $e^{-}+M_{2}^{+} \rightarrow$ $M^{*}+M$. The corresponding cross-section could be as high as $10^{-13}-10^{-14} \mathrm{~cm}^{2}$ at room temperature but it decreases by $2-3$ orders of magnitude for $T_{e} \sim 1-10 \mathrm{eV}$ [17]. In the collision-assisted recombination 
process, the electron recombines on the parent ion with the assistance of a momentum conserving neutral atom or molecule: $e^{-}+M^{+}+M \rightarrow M+M$. For an electron density $n_{e}$ on the order of $10^{17} \mathrm{~cm}^{-3}$ (corresponding to the density in the peaks of interference fringes), and a neutral density $n_{a}=2.710^{19} \mathrm{~cm}^{-3}$, the dissociative recombination time should be on the order of a few ns, while the collision-assisted recombination time should be in the subnanosecond range. Therefore the latter process dominates under our experimental conditions. Indeed, several authors have already discussed the decay of plasma generated by laser filamentation in air in terms of predominant collision-assisted recombination $[12,13]$.

The electron density evolution in the zone of the filament crossing can be described by the following equation

$$
\frac{\partial \mathrm{n}_{\mathrm{e}}}{\partial \mathrm{t}}=\mathrm{n}_{\mathrm{a}} \sigma_{\mathrm{M}}\left[2 \mathrm{I}_{0}(1+\cos (\mathrm{qz}))\right]^{\mathrm{M}}+\mathrm{D}_{\mathrm{am}} \frac{\partial^{2} \mathrm{n}_{\mathrm{e}}}{\partial \mathrm{z}^{2}}-\beta \mathrm{n}_{\mathrm{e}}^{2}
$$

where $\beta=K n_{a}$ is the coefficient of electron recombination, $q=2 \pi / \Lambda$ is the main grating period, $I_{0}$ is the laser intensity in the filament, and $\sigma_{M}$ is an effective cross section of strong field ionization. Here, we assume the plasma quasi-neutrality (the grating period is much larger than the electron mean free path and the electron Debye length) and weak ionization $\left(n_{e} \ll n_{a}\right)$. The parameter M specifies the dependence of the ionization rate on the laser intensity. It is different from just a number of photons needed for ionization as it is supposed by the simple multiphoton ionization model. We use instead the power M extracted from the PPT (Perelomov, Popov, and Terent'ev) theory, which has been shown by Chin to describe correctly ionization of gases in the relevant intensity range [18, 19]. We extract an effective number of simultaneously absorbed photons which varies from $M=4$ for $\mathrm{Kr}$ to $M=7$ for $\mathrm{N}_{2}$ [18]. The plasma grating profile created by the multi-photon ionization is therefore highly anharmonic, it contains a series of harmonics $N_{q}$ with $N \leq M$. The variation of the refraction index of the plasma, $\Delta N=-n_{e} / 2 n_{c r}$, is directly proportional to the ratio of the electron density to the plasma critical density, $n_{c r}=\varepsilon_{0} m_{e} \omega_{0}^{2} / e^{2}$, so that the grating evolution deduced from Eq. (1) contains $M$ spatial harmonics. 
As the laser pulse duration is much shorter than the grating relaxation time, we solve this equation in two steps. First we calculate the electron density distribution after the end of the laser pulse, while neglecting the two last terms in the right hand side. The electron density reads: $n_{e 0}(z)=n_{0}(1+\cos (q z))^{M}$, where $n_{0}=2^{M} n_{a} \sigma_{M} \int I_{0}^{M} d t$. In a second step, we solve the equation for $n_{e}$ by considering only the last two terms of Eq. (1) with the function $\mathrm{n}_{\mathrm{e} 0}(\mathrm{z})$ as the initial condition.

In atomic gases, the average electron density decays relatively slowly. In Ar, the grating amplitude corresponding to the main harmonic decreases by a factor 2 in 1 ns while the second harmonic $q_{2}=2 q$ requires less than $400 \mathrm{ps}$. This is the consequence of the fact that the rate of ambipolar diffusion rate increases as the square of the harmonic number. In molecular gases, the plasma recombination time is much shorter, comparable to the decay time of the grating measured in configuration A, so that both recombination and diffusion play a role in the relaxation of the plasma grating.

To find plasma parameters $D_{a m}, \beta$, and the initial electron density $n_{0}$, the measured grating decay curves for the three measured angles are best fitted for each gas. The initial conditions for solving Eq. (1) are obtained from fringe contrast measurements at different delays in configuration B, as discussed in details in reference [12]. Figure 3(a) shows an example of the far field image of the diffracted probe beam in $\mathrm{O}_{2}$ measured at a probe delay of 4.5 ps. From a best fit of the fringe profile across coordinate $x$ at maximum contrast at a given delay, the electron density at the corresponding time can be extracted. Then by changing the delay of the probe beam $\tau$, the decay of the plasma is obtained, allowing extraction of the coefficient $\beta$ and by extrapolation the initial density $n_{0}$. Typically the initial electron density is on the order of $10^{17}-$ $10^{18} \mathrm{~cm}^{-3}$. This value is in agreement with that calculated using the PPT theory $[18,19]$, under the assumption of a complete interference between the two crossing laser fields. The numerical fitting procedure is stringent for molecular gases, since for a given set of parameters $D_{a m}$ and $\beta$, the diffraction patterns in configuration A and B as well as their time dependence must be reproduced. Extracted values of $\beta$ and $D_{a m}$ are given in Table 1 for several atomic and molecular gases. There is good agreement between 
diffusion coefficients $D_{a m}$ from our experiments and ambipolar diffusion coefficients $D_{a}$ extracted from mobility measurements. To calculate $D_{a}$ we use $T_{i}=300 \mathrm{~K}, T_{e}=0.5 \mathrm{eV}$, typical of filaments [20] and $\mu_{i}$ values are taken from the literature [21].

The constant $K$ appearing in the recombination coefficient was determined at a very low pressure and at $300 \mathrm{~K}$ in $\mathrm{He}$ [22] $\left(10^{-26}\right.$ to $\left.10^{-27} \mathrm{~cm}^{6} / \mathrm{s}\right), \mathrm{O}_{2}$ [23] $\left(K \sim 10^{-30} \mathrm{~cm}^{6} / \mathrm{s}\right)$, and $\mathrm{Ne}\left(K \sim 10^{-27} \mathrm{~cm}^{6} / \mathrm{s}\right)$ [24]. The present experiment gives for the first time a value of $K$ for several gases at atmospheric pressure.

From Table 1, one can see that atomic gases have a much lower recombination rate than molecular gases. We note however that $\mathrm{Ne}$ recombines faster than other atomic gases. We attribute this efficient dissociative recombination to rapid dimer formation. The extracted dissociation coefficient is consistent with the value reported in Ref. [24].

To conclude, we have studied the dynamics of the plasma grating created by two intercepting filaments in different gases. The grating fringe evolution is dominated by ambipolar diffusion in atomic gases and by a combination of diffusion and recombination in molecular gases. The study of the plasma grating evolution provides a simple technique to determine several characteristics of this unusual type of plasma which is dense but weakly ionized.

We acknowledge fruitful discussions with Dr B. Forestier, technical help from J. Carbonnel, A. Dos Santos and Dr J. Gautier and financial support from DGA. 


\section{References}

[1] V. I. Talanov, "Self-focusing of wave beams in non linear media”, Radiophysics 9, 138-141 (1965)

[2] P. L. Kelley, "Self-Focusing of Optical Beams", Phys. Rev. Lett. 15, 1005 (1965)

[3] A. Couairon and A. Mysyrowicz, "Femtosecond filamentation in transparent media", Phys. Rep. 441, 47-189 (2007)

[4] A. C. Bernstein, M. McCormick, G. M. Dyer, J. C. Sanders, and T. Ditmire, “Two-Beam Coupling between Filament-Forming Beams in Air", Phys. Rev. Lett. 102, 123902 (2009)

[5] Y. Liu, M. Durand, S. Chen, A. Houard, B. Prade, B. Forestier, and A. Mysyrowicz, "Energy Exchange between Femtosecond Laser Filaments in Air”, Phys. Rev. Lett., 105, 055003 (2010)

[6] X. Yang, J. Wu, Y. Peng, Y. Tong, P. Lu, L. Ding, Z. Xu, and H. Zeng, "Plasma waveguide array induced by filament interaction", Opt. Lett. 34, 3806-3008 (2009)

[7] X. Yang, J. Wu, Y. Tong, L. Ding, Z. Xu, and H. Zeng, "Femtosecond laser pulse energy transfer induced by plasma grating due to filament interaction in air”, Appl. Phys. Lett. 97, 071108 (2010)

[8] S. Suntsov, D. Abdollahpour, D. G. Papazoglou, and S. Tzortzakis, "Femtosecond laser induced plasma diffraction gratings in air as photonic devices for high intensity laser applications", Appl. Phys. Lett. 94, 251104 (2009)

[9] Y. Liu, M. Durand, A. Houard, B. Forestier, A. Couairon, A. Mysyrowicz, "Efficient generation of third harmonic radiation in air filaments: A revisit", Opt. Commun. 284, 4706-4713 (2011)

[10] M. Durand, Y. Liu, B. Forestier, A. Houard, and A. Mysyrowicz, "Experimental observation of a travelling plasma grating formed by two crossing filaments in gases”, Appl. Phys. Lett., 98, 121110 (2011)

[11] L. Shi, W. Li, Y. Wang, X. Lu, L. Ding, and H. Zeng, “Generation of High-Density Electrons Based on Plasma Grating Induced Bragg Diffraction in Air”, Phys. Rev. Lett. 107, 095004 (2011)

[12] G. Rodriquez, A. R. Valenzuela, B. Yellampalle, M.J. Schmitt, and K.-Y. Kim, "In-line holographic imaging and electron density extraction of ultrafast ionized air filaments", J. Opt. Soc. Am. B, 25, 1988-1997 (2008)

[13] S. Tzortzakis, B. Prade, M. Franco, and A. Mysyrowicz, "Time-evolution of the plasma channel at the trail of a self-guided IR femtosecond laser pulse in air”, Opt. Commun. 181, 123-127 (2000)

[14] J. Wahlstrand, Y. Chen, Y. H Cheng, S. Varma, H. Milchberg, "Measurements of the high field optical nonlinearity and electron density in gases: Application to filamentation experiments", IEEE Journal of Quantum Electronics, 48, Issue 5 (2012) 
[15] The sharp dips observed after 3 ps and 5.8 ps for $\phi=90^{\circ}$ are due to spontaneous revivals of the refractive index [15] which diffract the probe pulse before it reaches the grating volume. For clarity, the dips observed at other angles $\phi$ have been removed.

[16] E. T. J. Nibbering, G. Grillon, M. A. Franco, B. S. Prade, and A. Mysyrowicz, "Determination of the inertial contribution to the nonlinear refractive index of air, $\mathrm{N}_{2}$, and $\mathrm{O}_{2}$ by use of unfocused highintensity femtosecond laser pulses", J. Opt. Soc. Am. B 14, 650-660 (1997)

[17] A. I. Florescu and J. B. A. Mitchell, "Dissociative recombination”, Phys. Rep. 430, 277 (2006)

[18] S. L. Chin, "From Multiphoton to Tunnel Ionization", Advances in Multiphoton Processes and Spectroscopy, S. H. Lin, A. A. Villaeys and Y. Fujimura, eds. (World Scientific, Singapore, 16, 249-272 (2004))

[19] A. M. Perelomov, V. S. Popov, and M. V. Terent'ev, “Ionization of atoms in an alternating electric field", Sov. Phys. JETP 23, 924-934 (1966)

[20] P. Sprangle, J. R. Peñano, B. Hafizi and C. A. Kapetanakos, "Ultrashort laser pulses and electromagnetic pulse generation in air and on dielectric surfaces”, Phys. Rev. E 69, 066415 (2004)

[21] E. U. Condon \& H. Odishaw, Handbook of physics, second edition, McGraw Hill (New York, 1967)

[22] J. Berlande, M. Cheret, R. Deloche, A. Gonfalone and C. Manus, "Pressure and electron density dependence of the electron-ion recombination coefficient in helium”, Phys. Rev. A 1, 3 (1970)

[23] L. M. Chanin, A. V. Phelps, and M. A. Biondi, "Measurement of the attachement of slow electrons in Oxygen”, Phys. Rev. Lett. 2, 344-346 (1959)

[24] R. N. Bhave and R. Cooper, "Two- and Three-body Ion-Electron Recombination Rate coefficients in Neon”, Aust. J. Phys. 48, 503-513 (1995) 
Figure 1:

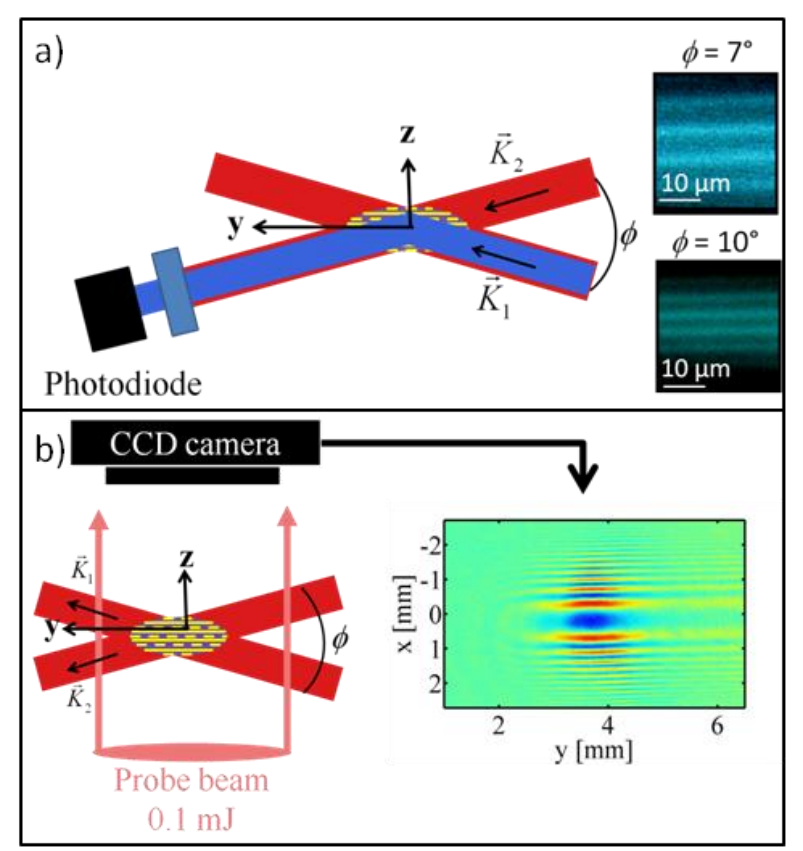


Figure 2:
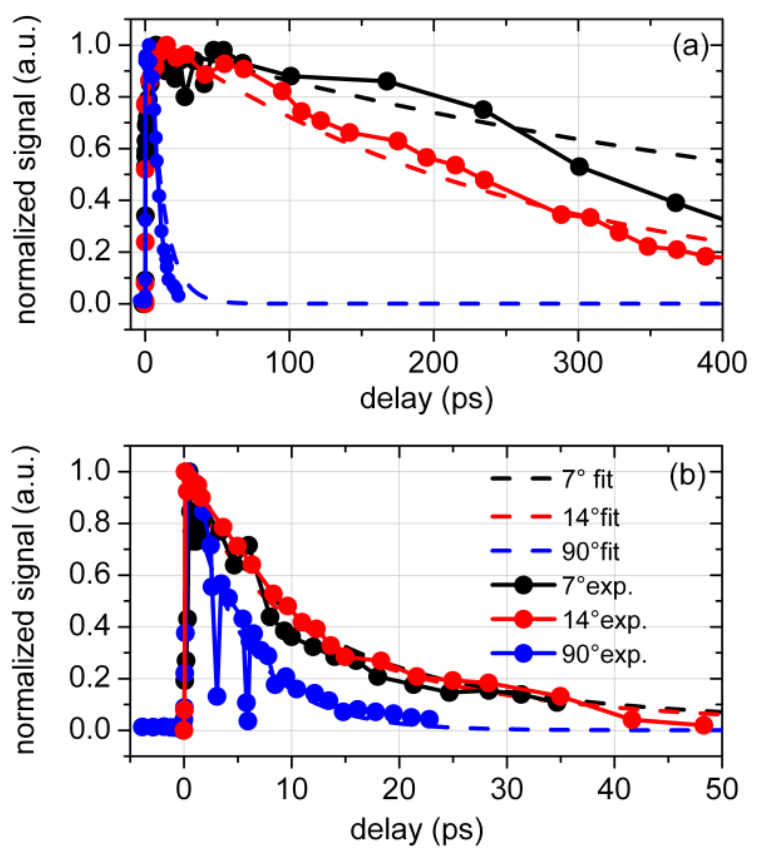
Figure 3:
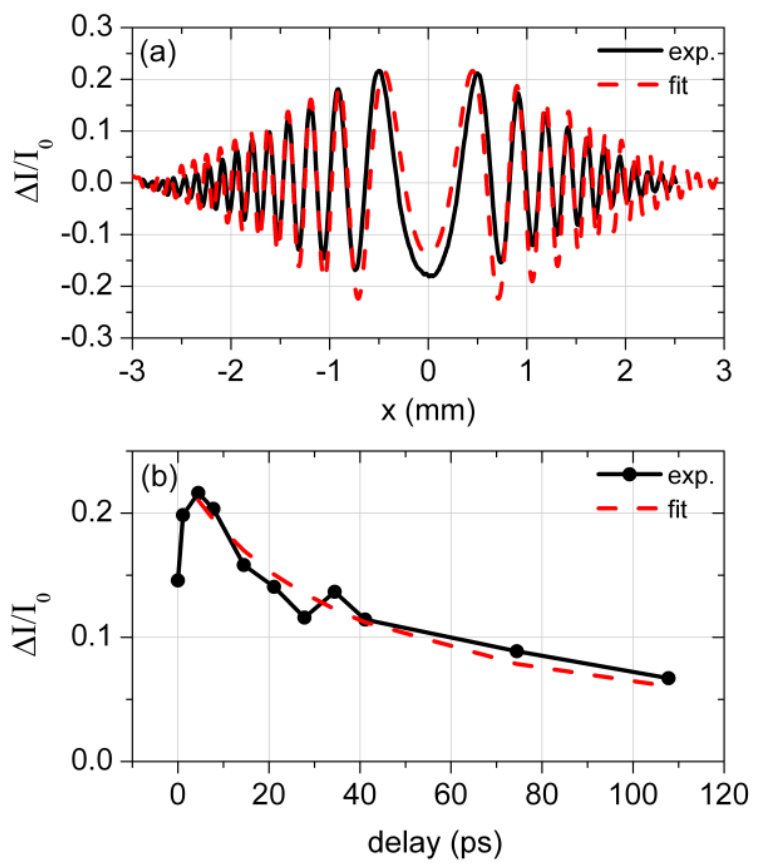
Table 1:

\begin{tabular}{ccccccccc}
\hline & $\mathbf{N e}$ & $\mathbf{A r}$ & $\mathbf{K r}$ & $\mathbf{X e}$ & $\mathbf{O}_{2}$ & $\mathbf{A i r}$ & $\mathbf{C O}_{2}$ & $\mathbf{N}_{2}$ \\
\hline$D_{a}=D_{i}\left(1+\frac{T_{e}}{T_{i}}\right)\left(\mathrm{cm}^{2} / \mathrm{s}\right)$ & 2.2 & 0.84 & 0.47 & 0.3 & 1.2 & 0.71 & 0.44 & 0.94 \\
$D_{a m}\left(\mathrm{~cm}^{2} / \mathrm{s}\right)$ & 1 & 0.84 & 0.52 & 0.3 & 1.1 & 0.25 & 0.44 & 0.6 \\
$n_{0}\left(\mathrm{~cm}^{-3}\right)$ & $7.4 \times 10^{17}$ & $3.3 \times 10^{18}$ & $1.8 \times 10^{18}$ & $8.7 \times 10^{17}$ & $1.2 \times 10^{18}$ & $1.3 \times 10^{18}$ & $9 \times 10^{17}$ & $1.3 \times 10^{18}$ \\
$\beta\left(\mathrm{cm}^{3} / \mathrm{s}\right)$ & $6.4 \times 10^{-9}$ & $1.8 \times 10^{-10}$ & $9.5 \times 10^{-10}$ & $1.7 \times 10^{-9}$ & $4.1 \times 10^{-8}$ & $5.3 \times 10^{-8}$ & $1.8 \times 10^{-7}$ & $3.3 \times 10^{-8}$ \\
$K\left(\mathrm{~cm}^{6} / \mathrm{s}\right)$ & $2.3 \times 10^{-28}$ & $6.6 \times 10^{-30}$ & $3.5 \times 10^{-29}$ & $6.3 \times 10^{-29}$ & $1.5 \times 10^{-27}$ & $2 \times 10^{-27}$ & $6.7 \times 10^{-27}$ & $1.2 \times 10^{-27}$ \\
\hline
\end{tabular}




\section{Figure Captions:}

FIG 1: (a) Pump-probe set up (configuration A) used to study the decay of the plasma grating. The probe pulse at $400 \mathrm{~nm}$ propagates collinearly with one filament and is diffracted along the other filament. The luminescence of the plasma in air is also shown for two incident angles showing the formation of a grating. (b) Top view of the holographic imaging set-up used to study the time evolution of the plasma (configuration B). The far field diffraction pattern of a probe pulse at $800 \mathrm{~nm}$ crossing the plasma area in $\mathrm{O}_{2}$ with a delay of $4.5 \mathrm{ps}$ is also shown.

FIG 2: Normalized intensity of diffracted probe signal as a function of the delay $\tau$ (ps) in (a) argon and (b) $\mathrm{O}_{2}$ for crossing angles $\Phi$ of $7^{\circ}, 14^{\circ}$ and $90^{\circ}$. Measurements performed in configuration A are represented by dots. Calculations are represented by continuous line.

FIG 3: (a) Far field diffraction pattern of the probe beam in configuration $\mathrm{B}$ in $\mathrm{O}_{2}$; fringes with maximum contrast are compared to calculations assuming a double Gaussian plasma profile. (b) Decay of the fringe contrast obtained in $\mathrm{O}_{2}$ from successive diffraction patterns.

Table 1: Plasma parameters determined from the dynamics of the plasma grating: the parameters $D_{a m}, n_{0}$ and $\beta$ are obtained from the best fitting the time dependence of the probe signal by the solutions of Eq. (1). The parameter $\mathrm{K}$ is calculated from the relation $\beta=\mathrm{K} \mathrm{n}$, and the ambipolar diffusion coefficient are given for a comparison by using the plasma parameters expected in the filament [20, 21]. 